\title{
Acceso a la justicia en materia ambiental en la Unión Europea*
}

\author{
Álvaro Sánchez Bravo**
}

\section{INTRODUCCIÓN}

El inicio de una política europea en materia de medio ambiente se remonta a la conferencia de Jefes de Estado y de Gobierno europeos celebrada en octubre de 1972, en la que se confirmó la necesidad de establecer una política común en este ámbito. Mediante el Acta Única Europea de 1987 se introdujo un nuevo título sobre medio ambiente en el Tratado de Roma, que constituyó el primer fundamento jurídico claro para la política comunitaria en materia de medioambiente. Posteriores revisiones de los Tratados reforzaron el compromiso de la Comunidad con la protección del medio ambiente y el papel del Parlamento Europeo en el desarrollo de la política medioambiental. El principal fundamento jurídico de la política medioambiental de la UE son hoy los artículos 191 a 193 del Tratado de Lisboa (anteriormente, artículos 174 a 176 del Tratado constitutivo de la Comunidad Europea, TCE). La adopción del Tratado de Lisboa comporta una sola modificación significativa de la política de la Unión en el ámbito del medio ambiente, a saber, la inclusión del "cambio climático", considerado un asunto de especial preocupación. Según el artículo 191, apartado 2, la política medioambiental de la UE se basa en los principios de cautela, acción preventiva, corrección de la contaminación en su fuente y "quien contamina paga".

Por otro lado, los procedimientos de infracción establecidos en los artículos $258^{1}$ y 260 del Tratado de Funcionamiento de la Unión Europea (TFUE), constituyen una potente herramienta para abordar los problemas de aplicación. Las denuncias relativas a la aplicación de la legislación medioambiental a menudo

\footnotetext{
* Texto revisado del trabajo publicado en A. Sánchez Bravo (Director), Democracia, Pluralismo y Derechos Humanos, Thomsom Reuters ARANZADI, Zizur Mayor, 2019.

** Doctor en Derecho. Profesor de la Facultad de Derecho de la Universidad de Sevilla. Director de los Encuentros Hispano-Brasileño de Filosofía del Derecho y Derechos Humanos. Presidente de la Asociación Andaluza de Derecho, Medio Ambiente y Desarrollo Sostenible. Expert European Research Council Executive Agency (ERCEA). European Commission. Expert Water Lex - UNO. Coeditor Revista Internacional de Direito Ambiental.
} 
adoptan la forma de preguntas escritas y peticiones dirigidas al Parlamento Europeo. Esto pone de manifiesto la preocupación de los ciudadanos de la UE por el estado del medio ambiente y los "resultados ecológicos" de los Estados miembros. En este contexto, es necesario desarrollar nuevos métodos de trabajo junto con los Estados miembros en todas las fases del ciclo de transposición legislativa.

Más del $75 \%$ de los ciudadanos europeos consideran que la legislación medioambiental de la UE es necesaria para proteger el medio ambiente en su país, y casi el $80 \%$ está de acuerdo en que las instituciones de la UE deben tener la posibilidad de comprobar si esa legislación se está aplicando correctamente en su país ${ }^{2}$.

La política y la legislación de medio ambiente de la UE aportan ventajas indiscutibles: protegen, conservan y mejoran el medio ambiente para las generaciones actuales y futuras y preservan la calidad de vida de los ciudadanos de la UE. Cuando su aplicación es deficiente se generan elevados costes sociales, económicos y medioambientales y se crean condiciones de competencia desiguales para las empresas. La importancia de la correcta ejecución del acervo de la UE en materia de medio ambiente también se refleja en el Séptimo Programa de Medio Ambiente ${ }^{3}$.

La legislación ambiental de la Unión Europea incluye la legislación referente al logro de los siguientes objetivos de la política de la UE sobre medio ambiente $^{4}$, establecidos en el art. 191TFUE:

- conservación, protección y la mejora de la calidad del medio ambiente, la protección de la salud de las personas,

- la utilización prudente y racional de los recursos naturales, así como

- $\quad$ el fomento de medidas a escala internacional destinadas a hacer frente a los problemas regionales del medio ambiente, $\mathrm{y}$, en particular, a luchar contra el cambio climático.

La conservación, protección y mejora medio ambiental supone un valor compartido por los europeos que impone, en el ámbito legislativo, un marco común de obligaciones para las autoridades públicas y de derechos para el público.

Para ello es necesario que se reconozca en el ámbito medioambiental, como hace la legislación europea, el acceso a la justicia que debe reflejar los derechos públicos afectados. No podía ser de otra manera en una estructura jurídico-política, como la de la UE, fundamentada en el Estado de Derecho, que incluye una tutela judicial efectiva de los derechos conferidos por el Derecho de la UE. Así los establece el art. 19.1 del Tratado de la Unión Europea (TUE) al establecer que "los Estados Miembros establecerán las vías de recurso necesarias para a garantizar la tutela judicial efectiva en los ámbitos cubiertos por el derecho de la Unión"5. 
A mayor abundamiento, la tutela judicial efectiva está directamente vinculada a una interpretación uniforme del Derecho de la UE por parte del Tribunal de Justicia de la Unión Europea (TJUE), y la posibilidad, cuando no, obligación, de los que los jueces y tribunales nacionales planteen preguntas relativas a la validez e interpretación de actos de las instituciones y órganos de la UE al TJUE mediante cuestiones prejudiciales, conforme al art. 267 TFUE$^{6}$. Ahora bien, si el acceso a la justicia nacional no es posible o es excesivamente difícil, el mandato comunitario sería inútil.

Por tanto, el acceso a la justicia en materia medioambiental es intrínseco a la legislación medioambiental de la UE y descansa en principios fundamentales del derecho de la UE reflejados en las disposiciones de los Tratados de la Unión, el Convenio de Aarhus ${ }^{7}$ y el Derecho derivado interpretado en la jurisprudencia del TJUE.

Más allá del margen de aplicación del Derecho derivado, las actuales disposiciones legislativas de los Estados Miembros en materia de acceso a la justicia en materia ambiental difieren considerablemente ${ }^{8}$, siendo las deficiencias de aplicación más acuciantes las referentes a la gestión de residuos, naturaleza y biodiversidad, calidad del aire, y gestión y calidad del agua.

En esta materia, se identificaron una serie de problemas:

- los ciudadanos y las ONG se ven obstaculizadas al acceder a los órganos jurisdiccionales nacionales. Esto provoca una avalancha de cuestiones prejudiciales ante en el TJUE. Igualmente, los ciudadanos se ven indirectamente afectados cuando la falta de acceso a la justicia impide el control de la aplicación de la normativa ambiental por parte de las administraciones públicas.

- Las administraciones públicas y los órganos jurisdiccionales nacionales asumen pesadas cargas derivadas de largos procesos relacionados con el acceso a la justicia.

- Para las empresas, los retrasos en la toma de decisiones administrativas, referentes a dilatados litigios, influyen negativamente; especialmente las pequeñas y medianas empresas. Igualmente pueden verse afectadas cuando el acceso ineficaz a la justicia determina que no pueda acceder al medio ambiente limpio del que dependen, o cuando los gobiernos no realicen inversiones favorables para la economía verde.

Es por ello que la efectivación del derecho de los ciudadanos al medio ambiente no sería posible sin un reconocimiento, y reforzamiento, del acceso del público a la información relativa al medio ambiente, en su participación y acceso a la justicia en este ámbito, y en la difusión de la información, contribuyendo de este modo a ajustar la legislación de la UE, dando cumplimiento al Convenio de Aarhus. 


\section{EL CONVENIO DE AARHUS}

El Convenio de la Comisión Económica para Europa de Naciones Unidas sobre acceso a la información pública, participación pública en la toma de decisiones y el acceso a la justicia en materia de medio ambiente (Convenio Aarhus), fue elaborado el 25 de junio de 1998, estableciendo, en su Preámbulo que: "para que los ciudadanos puedan disfrutar del derecho a un medio ambiente saludable y cumplir el deber de respetarlo y protegerlo, deben tener acceso a la información medioambiental, estar legitimados para participar en los procesos de toma de decisiones y tener derecho de acceso a la justicia cuando tales derechos sean denegados".

La Unión Europea, entonces Comunidad Europea, aprobó en 2005, mediante una Decisión ${ }^{9}$, el Convenio de Aarhus, que fue firmado por la Comunidad Europea y sus Estados miembros en 1998.

El Convenio, vigente desde el 30 de octubre de 2001, parte de la idea de que una mayor implicación y sensibilización de los ciudadanos con relación a los problemas medioambientales conduce a una mejor protección del medio ambiente. El Convenio tiene por objeto contribuir a proteger el derecho de cada persona, de las generaciones presentes y futuras, a vivir en un medio ambiente adecuado para su salud y su bienestar. Para alcanzar dicho objetivo, el Convenio propone intervenir en tres ámbitos:

- garantizar el acceso del público a las informaciones sobre medio ambiente de que disponen las autoridades públicas;

- favorecer la participación del público en la toma de decisiones que tengan repercusiones sobre el medio ambiente;

- ampliar las condiciones de acceso a la justicia en materia de medio ambiente.

Las instituciones comunitarias responden a la definición de autoridad pública del Convenio, en igualdad de condiciones que las autoridades nacionales o locales.

Las Partes del Convenio se comprometieron a aplicar las disposiciones enumeradas, por lo que deben:

- adoptar las medidas legales, reglamentarias o de otro tipo necesarias;

- procurar que los funcionarios y las autoridades públicas ayuden y aconsejen a los ciudadanos para tener acceso a la información, participar en el proceso de toma de decisiones y recurrir a la justicia;

- favorecer la educación ecológica del público y concienciarlo respecto de los problemas medioambientales; 
- conceder su reconocimiento y apoyo a las asociaciones, organizaciones o grupos que tengan por objeto la protección del medio ambiente.

El primer pilar del citado Convenio, que hace referencia al acceso del público a la información, fue aplicado a escala comunitaria mediante la Directiva 2003/4/CE relativa al acceso del público a la información medioambiental ${ }^{10}$. El segundo pilar, relativo a la participación del público en los procedimientos medioambientales, fue transpuesto mediante la Directiva 2003/35/CE ${ }^{11}$. Por último, un Reglamento ${ }^{12}$ pretende garantizar la aplicación de las disposiciones y principios del Convenio a las instituciones y órganos comunitarios.

\section{ACCESO A LA JUSTICIA EN MATERIA AMBIENTAL}

En materia de acceso a la justicia, el Convenio de Aarhus, establece que el público podrá recurrir a la justicia en condiciones adecuadas, en el marco de la legislación nacional, cuando considere que sus derechos en materia de acceso a la información (solicitud de información ignorada, rechazada de forma abusiva o tenida en cuenta de forma insuficiente) han sido vulnerados.

Se garantiza asimismo el acceso a la justicia en caso de violación del procedimiento de participación previsto en el Convenio. Se admite, además, el acceso a la justicia para la solución de controversias vinculadas con actos u omisiones de particulares o de autoridades públicas que infrinjan las disposiciones medioambientales del ordenamiento jurídico nacional.

En el marco comunitario europeo, el primer intento de regulación de esta materia fue una propuesta de Directiva publicada en octubre de $2003^{13}$ con el fin de transponer el tercer pilar del Convenio, que garantiza el acceso del público a la justicia en materia de medio ambiente, pero nunca pasó de la fase de primera lectura en el Parlamento Europeo. Esta institución deseaba que la Directiva estableciera un marco mínimo para el acceso a la justicia en materia de medio ambiente y que los Estados miembros tuvieran libertad para conceder un acceso mayor. Propuso algunas enmiendas que ampliarían el acceso a la justicia en este ámbito a las organizaciones de ciudadanos enfrentados a un problema ecológico concreto, y no limitado, como en la propuesta original, a las entidades medioambientales.

Tras considerar diversas opciones, y tras 14 años de espera, la Comisión Europea decidió que la mejor y más adecuada forma de abordar esta cuestión será mediante la elaboración de una Comunicación ${ }^{14}$ interpretativa sobre la justicia en materia medioambiental, justificada en que 
"al reunir la abundante jurisprudencia existente del TJUE, y sacar cuidadosas conclusiones a partir de ella, se aporta una gran claridad y una fuente de referencia a las siguientes entidades: las administraciones naciones responsables de garantizar la correcta aplicación de la legislación medioambiental de la UE; los órganos jurisdiccionales nacionales, que garantizan el respeto de la legislación de la UE y que tienen competencias para plantear preguntas sobre la validez e interpretación de dicha legislación al TJUE; el público, en concreto, las personas y las ONG de protección del medio ambiente, que ejercen una función de defensa de los intereses públicos; y los operadores económicos, que comparten un interés en que la aplicación de la legislación sea previsible. El procedimiento de adopción simplificado ayudaría a la Comisión a emprender una iniciativa efectiva a corto plazo"15.

Su ámbito de aplicación viene determinado por el acceso a la justicia en relación con decisiones, actos y omisiones de autoridades públicas de los Estados miembros, no aplicándose a litigios entre particulares, ni al control jurisdiccional de los actos de las instituciones de la UE. Dentro de estas propias limitaciones, dimanantes de la forma jurídica elegida por la Comisión para regular estas cuestiones $^{16}$, se pretende aclarar como el público puede ampararse en los órganos jurisdiccionales nacionales, los cuales se encuentran en una mejor posición para identificar soluciones más adecuadas, al estar más cercanos a los hechos y el contexto en que se producen ${ }^{17}$.

Siguiendo lo establecido por la propia Comunicación, desglosaremos los contenidos que garantizarán el acceso a la justicia medioambiental en la Unión europea.

1. Intereses públicos, obligaciones y derechos pertinentes para el ejercicio de la tutela judicial.

El acceso a la justicia medioambiental, como señaló el TJUE ${ }^{18}$, representa un conjunto de derechos auxiliares que sirve a dos propósitos: permite a las personas y asociaciones ejercer el derecho que les otorga la legislación de la UE, y ayudar a garantizar el cumplimiento de los objetivos y obligaciones de la legislación medio ambiental.

La legislación medioambiental de la UE pretende garantizar intereses generales como un aire limpio, recursos hídricos seguros y adecuados y un buen estado de la biodiversidad. La participación activa del público supone un interés público medioambiental concomitante que apoya dichos intereses.

Por tanto, los intereses públicos y de otros tipos dispuestos en la legislación medioambiental de la UE y las obligaciones conexas impuestas a las autoridades públicas dan lugar a derechos procesales y sustantivos de los individuos y sus asociaciones, que deben ser protegidos por los órganos jurisdiccionales nacionales. 
En este ámbito, las ONG de protección medioambiental desempeñan un papel importante a la hora de garantizar el cumplimiento de las obligaciones de la legislación medioambiental de la UE y disfrutan de amplios derechos para proteger el medio ambiente que los órganos jurisdiccionales nacionales deben defender. Por su parte, esa misma legislación confiere derechos sustantivos y procesales a las personas, referidos en particular a las obligaciones de las autoridades públicas de seguir un procedimiento correcto destinado a implicar al público, así como a las disposiciones en materia de salud de las personas y propiedad. ${ }^{19}$

\section{Legitimación Activa.}

La legitimación activa es el derecho a presentar un recurso judicial ante un órgano judicial u otro organismo independiente e imparcial a fin de proteger un derecho o interés del demandante relacionado con la legalidad de una decisión, acto u omisión de una autoridad pública. La legitimación activa puede variar dependiendo del objeto de la decisión, acto u omisión impugnada. Asimismo, puede variar dependiendo de si el demandante es una persona o una ONG de protección del medio ambiente reconocida.

Cualquier persona física o jurídica que presente una solicitud de información dispone de legitimación activa para impugnar una decisión, acto u omisión de la autoridad pública responsable de tramitar dicha solicitud. Los derechos a recibir información a través de la difusión activa también pueden permitir a las personas y las asociaciones presentar recursos judiciales.

Los requisitos de participación del público que se aplican a determinadas actividades concretas que pueden afectar al medio ambiente, confieren derechos a las personas afectadas y permiten a estas solicitar una revisión judicial de la decisión, acto u omisión en cuestión. En este ámbito, en el caso de las personas, la condición previa de tener que mostrar la "lesión de un derecho"; o un interés suficiente a fin de obtener legitimación activa para presentar un recurso en relación con una actividad concreta debe interpretarse y aplicarse a la luz de la obligación de conceder un amplio acceso a la justicia en materia medioambiental. Los derechos que pueden ser lesionados incluyen los derechos procesales de las personas que derivan de la legislación medioambiental de la UE (por ejemplo, derechos de participación del público) así como derechos sustantivos conferidos a la persona (por ejemplo, protección de la salud de las personas, derechos de propiedad). Respecto a las ONG, no deben establecerse requisitos excesivamente difíciles de satisfacer para obtener legitimación activa, debiendo prestarse especial atención a las pequeñas ONG locales. Igualmente, no deben establecerse criterios discriminatorios respecto a las ONG extranjeras. Igualmente, no debe obviarse que, aunque no posean legitimación activa de lege, determinadas asociaciones, organizaciones y grupos pueden disfrutar de legitimación activa de la misma forma que las personas, conforme al derecho nacional de cada uno de los Estados. ${ }^{20}$ 


\section{Alcance del Control Jurisdiccional.}

El alcance del control jurisdiccional determina cómo evaluarán los órganos jurisdiccionales nacionales la legalidad de las decisiones, los actos y las omisiones que se impugnen. Tiene dos componentes. El primero de ellos está relacionado con los posibles fundamentos del control jurisdiccional, es decir, los órdenes jurisdiccionales y las consideraciones de derecho que pueden invocarse. El segundo se refiere a la intensidad del control (o criterios de control).

Respecto a los fundamentos del control jurisdiccional deviene especialmente importante en aquellas jurisdicciones que únicamente consideran legítimas las denuncias en aquellos casos en los que se ha producido una vulneración de los derechos del demandante. En estos casos, los posibles fundamentos del control jurisdiccional suelen limitarse a las disposiciones jurídicas en las que se prevén los derechos individuales que sustentan la legitimación procesal que se haya invocado. Este aspecto también es importante en el caso de las restricciones cuyo objetivo es obligar a los demandantes a utilizar exclusivamente argumentos planteados en procedimientos administrativos previos (preclusión), o evitar que los demandantes abusen de los procesos judiciales al realizar alegaciones jurídicas irrelevantes.

Respecto a las actividades específicas sujetas a requisitos de participación pública, $\mathrm{y}$ en lo tocante a las personas físicas, cuando un Estado miembro condicione la admisibilidad de los recursos judiciales presentados por personas físicas a la vulneración de un derecho individual, dicho Estado también está autorizado a prever que la anulación de una decisión administrativa por parte de un órgano jurisdiccional nacional requiera la violación de un derecho individual del demandante. Por su parte, las ONG medioambientales reconocidas no están sujetas a limitaciones en cuanto a los motivos de derecho que pueden aducir, y pueden invocar todas las disposiciones de la legislación ambiental de la UE.

En lo relativo a la preclusión, el alcance del control llevado a cabo por un órgano jurisdiccional nacional no podrá quedar limitado a las objeciones que ya se hayan presentado dentro del plazo concedido en el procedimiento administrativo. No obstante, los órganos jurisdiccionales pueden declarar la inadmisibilidad de argumentos presentados que resulten abusivos o de mala fe.

Pero no vale ni es admisible cualquier tipo de control jurisdiccional, por el contrario, los Estados miembros deben garantizar un control jurisdiccional efectivo de la legalidad tanto del fondo como del procedimiento de cualquier decisión, acto u omisión que se incluya en el ámbito de aplicación de estas disposiciones, incluso cuando el Derecho derivado de la UE no incluya referencias explícitas a un criterio de control que abarque estos dos aspectos de la legalidad. Cuando resulte necesario, debe ampliarse el control a la legalidad de actos legislativos y reglamentarios en caso de que reduzcan o vulneren derechos procesales y sustantivos. ${ }^{21}$ 
4. Tutela Judicial Efectiva.

Como requisito general, todos los organismos de un Estado miembro deben eliminar las consecuencias ilícitas de las vulneraciones de la legislación ambiental de la UE. Los Estados miembros también deben abstenerse de tomar medidas que puedan suponer un peligro grave para el logro de un resultado previsto en la legislación ambiental de la UE.

A tenor del principio de autonomía procesal de los Estados miembros, corresponde al ordenamiento jurídico interno de cada Estado miembro determinar la regulación procesal concreta aplicable, siempre que tal regulación no sea menos favorable que la aplicable a situaciones similares de carácter interno (principio de equivalencia) y no haga imposible en la práctica o excesivamente difícil el ejercicio de los derechos conferidos por el ordenamiento jurídico de la UE (principio de efectividad).

La tutela judicial efectiva puede decaer en los casos de vicios procedimentales leves o irrelevantes, siempre que pueda demostrarse, sin coste alguno para la parte que solicite el control judicial de esos vicios, que los mismos no fueron relevantes para la toma de decisión impugnada.

Por otro lado, la tutela judicial se garantizará en las jurisdicciones nacionales cuando las mismas dispongan de mecanismos para adoptar medidas generales o específicas, incluidas las provisionales, ante incumplimientos de la legislación ambiental de la UE, y que pueden consistir en la suspensión, revocación o anulación de las decisiones o actos ilícitos, así como en los supuestos de inaplicación de actos legislativos o reglamentarios.

Pero estas medidas no serían eficaces, si las mismas no vinieran acompañadas de medidas para reparar los daños ilícitos causados, que deben abarcar, tanto la compensación por los daños económicos, como la reparación por los daños ilícitos por el medio ambiente. ${ }^{22}$

\section{Costas Procesales.}

Los Estados miembros deben garantizar que los procedimientos de control jurisdiccional no tengan un coste prohibitivo, que impida la impugnación de un asunto ante un órgano jurisdiccional nacional. A este respecto, el requisito de que el costo de los procedimientos de control jurisdiccional no sea prohibitivo está sujeto a interpretación a escala de la UE. Está relacionado con todos los costes que genera la participación en un procedimiento, incluidas las garantías financieras que debe presentar el demandante, y resulta de aplicación para todas las fases del proceso judicial. Los demandantes tienen derecho a una previsibilidad razonable en lo relativo a los gastos procesales a los que se exponen. En aquellos casos en los que el órgano jurisdiccional nacional esté capacitado para determinar el costo que deberá asumir un demandante cuyas alegaciones hayan sido desestimadas, podrían tenerse en cuenta cuestiones subjetivas relacionadas con el demandante, 
al tiempo que se garantiza que los gastos procesales no son objetivamente desmesurados. ${ }^{23}$

\section{CONCLUSIONES}

La regulación del acceso a la justicia ambiental constituía una regulación inaplazable. Si bien, el instrumento jurídico utilizado carece de la fuerza de obligar inherente a otros productos normativos comunitarios, al menos aporta elementos interpretativos que permite configurar un marco uniforme para el acceso a la justicia en materia medioambiental.

Máxime en un momento en que la Unión europea se encuentra en la encrucijada de reforzar el Estado de derecho ${ }^{24}$, ante el euroesceptiscismo, la vuelta de los nacionalismos exacerbados y el deterioro de la posición de la UE en la geopolítica mundial.

La política ambiental comunitaria sólo pude ser adecuada y eficaz si está basada, no sólo en el conocimiento científico y en los procedimientos técnicos, sino en una "democracia ambiental"; en el reconocimiento del derecho de los ciudadanos europeos a participar, cuestionar e impugnar aquellas políticas y decisiones que les afectan, también en el ámbito ambiental.

La Europa de los ciudadanos, y el derecho de acceso a la justicia, junto a los derechos de acceso a la información y participación deben ser manifestación de compromiso democrático manifestado en su texto fundacional.

Debemos seguir, por tanto, reivindicando el estatuto ciudadano nuestro derecho a ser oídos y a participar. En una Europa sumida en la crisis, no sólo económica, sino de valores, no queda otra solución que la profundización en la democracia real, en la recuperación del papel de los ciudadanos en el proceso de reconstrucción europea, en la bandera de la dignidad del ser humano frente a la economía.

\section{NOTAS}

1 "Si la Comisión estimare que un Estado miembro ha incumplido una de las obligaciones que le incumben en virtud de los Tratados, emitirá un dictamen motivado al respecto, después de haber ofrecido a dicho Estado la posibilidad de presentar sus observaciones.

Si el Estado de que se trate no se atuviere a este dictamen en el plazo determinado por la Comisión, ésta podrá recurrir al Tribunal de Justicia de la Unión Europea”.

2 Comisión Europea (2014): Eurobarómetro especial n. ${ }^{\circ}$ 416, p. 26. COMISIÓN EUROPEA. Eurobarómetro especial n 416. Bruselas, 2014.

3 DOUE L 354. 28.12.2013.

4 Vid. Art. 37 Carta de los Derechos Fundamentales de la Unión Europea. "Protección del medio ambiente". En las políticas de la Unión se integrarán y garantizarán, conforme al principio de desarrollo sostenible, un nivel elevado de protección del medio ambiente y la mejora de su calidad. 
5 Vid. Art. 47, Carta de los Derechos Fundamentales de la Unión Europea. Derecho a la tutela judicial efectiva y a un juez imparcial. "Toda persona cuyos derechos y libertades garantizados por el Derecho de la Unión hayan sido violados tiene derecho a la tutela judicial efectiva respetando las condiciones establecidas en el presente artículo. Toda persona tiene derecho a que su causa sea oída equitativa y públicamente y dentro de un plazo razonable por un juez independiente e imparcial, establecido previamente por la ley. Toda persona podrá hacerse aconsejar, defender y representar. Se prestará asistencia jurídica gratuita a quienes no dispongan de recursos suficientes siempre y cuando dicha asistencia sea necesaria para garantizar la efectividad del acceso a la justicia.”

6 "El Tribunal de Justicia de la Unión Europea será competente para pronunciarse, con carácter prejudicial:

a) sobre la interpretación de los Tratados;

b) sobre la validez e interpretación de los actos adoptados por las instituciones, órganos u organismos de la Unión.

Cuando se plantee una cuestión de esta naturaleza ante un órgano jurisdiccional de uno de los Estados miembros, dicho órgano podrá pedir al Tribunal que se pronuncie sobre la misma, si estima necesaria una decisión al respecto para poder emitir su fallo.

Cuando se plantee una cuestión de este tipo en un asunto pendiente ante un órgano jurisdiccional nacional cuyas decisiones no sean susceptibles de ulterior recurso judicial de derecho interno, dicho órgano estará obligado a someter la cuestión al Tribunal.

Cuando se plantee una cuestión de este tipo en un asunto pendiente ante un órgano jurisdiccional nacional en relación con una persona privada de libertad, el Tribunal de Justicia de la Unión Europea se pronunciará con la mayor brevedad."

7 The United Nations Economic Commission for Europe (UNECE). Convention on Access to Information, Public Participation in Decision-Making and Access to Justice in Environmental Matters.

8 COMUNICACIÓN DE LA COMISIÓN AL PARLAMENTO EUROPEO, AL CONSEJO, AL COMITÉ ECONÓMICO Y SOCIAL EUROPEO Y AL COMITÉ DE LAS REGIONES. Revisión de la aplicación de la normativa medioambiental de la UE: problemas comunes y cómo combinar esfuerzos para obtener mejores resultados. COM (2017) 63. Bruselas 03.02.2017.

9 Decisión 2005/370/CE del Consejo, de 17 de febrero de 2005, sobre la celebración, en nombre de la Comunidad Europea, del Convenio sobre el acceso a la información, la participación del público en la toma de decisiones y el acceso a la justicia en materia de medio ambiente. DOUE L 124. 17.05.2005.

10 Directiva 2003/4/CE, del Parlamento Europeo y del Consejo, de 28 de enero de 2003, relativa al acceso del público a la información medioambiental y por la que se deroga la Directiva 90/313/CEE del Consejo. DOUE L 41. 14.02.2003.

11 DIRECTIVA 2003/35/CE DEL PARLAMENTO EUROPEO Y DEL CONSEJO, de 26 de mayo de 2003, por la que se establecen medidas para la participación del público en la elaboración de determinados planes y programas relacionados con el medio ambiente y por la que se modifican, en lo que se refiere a la participación del público y el acceso a la justicia, las Directivas 85/337/CEE y 96/61/CE del Consejo. DOUE L 156. 25.06.2003.

12 Reglamento (CE) no 1367/2006, del Parlamento Europeo y del Consejo, de 6 de septiembre de 2006, relativo a la aplicación, a las instituciones y a los organismos comunitarios, de las disposiciones del Convenio de Aarhus sobre el acceso a la información, la participación del público en el proceso de toma de decisiones y el acceso a la justicia en materia de medio ambiente. DOUE L 264. 25.09.2006.

13 Propuesta de Directiva del Parlamento Europeo y del Consejo, sobre el acceso a la justicia en materia de medio ambiente. COM (2003) 624. Bruselas. 24.10.2003.

14 Comunicación de la Comisión relativa al acceso a la justicia en materia medioambiental. DOUE C 275. 18.08.2017. 
15 Idem, p. 3.

16 Vid. GARDEÑES SANTIAGO, M. "Las Comunicaciones interpretativas» de la Comisión: Concepto y Valor normativo".

17 Idem, p. 4.

18 Asunto C-71/14 East Sussex, ap. 52, y Asunto C 72/95 Kraaijeveld, ap. 56.

19 Comunicación de la Comisión relativa al acceso a la justicia en materia medioambiental, cit. pp. 6-9.

20 Comunicación de la Comisión relativa al acceso a la justicia en materia medioambiental, cit. pp. 11-19.

21 Comunicación de la Comisión relativa al acceso a la justicia en materia medioambiental, cit. pp. 19-27.

22 Comunicación de la Comisión relativa al acceso a la justicia en materia medioambiental, cit. pp. 27-31.

23 Ídem, pp. 31-34.

24 Vid. Sánchez Bravo, A., "Afianzar el Estado de Derecho en la Unión europea”, en la obra colectiva, edición de A. Sánchez Bravo, Derechos Humanos, Ciudadanía y Globalización, PuntoRojo Libros, Sevilla, 2017, pp. 170-186.

\section{REFERÊNCIAS}

COMISIÓN DE LAS COMUNIDADES EUROPEAS. Propuesta de Directiva del Parlamento Europeo y del Consejo, sobre el acceso a la justicia en materia de medio ambiente. COM (2003) 624. Bruselas. 24.10.2003. Disponible en: http://ec.europa.eu/ transparency/regdoc/rep/1/2003/ES/1-2003-624-ES-F1-1.Pdf. Consulta en 06 de marzo de 2019.

COMISIÓN EUROPEA. Eurobarómetro especial n 416. Bruselas, 2014.

COMUNICACIÓN DE LA COMISIÓN AL PARLAMENTO EUROPEO, AL CONSEJO, AL COMITÉ ECONÓMICO Y SOCIAL EUROPEO Y AL COMITÉ DE LAS REGIONES. Revisión de la aplicación de la normativa medioambiental de la UE: problemas comunes y cómo combinar esfuerzos para obtener mejores resultados. COM (2017) 63. Bruselas 03.02.2017. Disponible en: https://eur-lex.europa.eu/legal-content/ ES/TXT/?uri=CELEX\%3A52017SC0042. Consulta en: 06 de marzo de 2019.

GARDEÑES SANTIAGO, M. "Las Comunicaciones interpretativas” de la Comisión: Concepto y Valor normativo”. Disponible en http://www.cepc.gob.es/Controls/Mav/ getData.hx?MAVqs= aWQ9MjgzMDImaWRIPTEwMzcmdXJsPTUmbmFtZT1SSUVf MDE5XzAwM18xNDUucGRmJmZpbGU9UklFXzAxOV8wMDNfMTQ1LnBkZiZ0YWJs YT1BcnRpY3VsbyZjb250ZW50PWFwcGxpY2F0aW9uL3BkZg==. Consulta 09.01.2018.

http://www.unece.org/fileadmin/DAM/env/pp/documents/cep43e. Consulta en: 06 de marzo de 2019. 
SÁNCHEZ BRAVO, A., "Afianzar el Estado de Derecho en la Unión europea”, en la obra colectiva, edición de A. Sánchez Bravo, Derechos Humanos, Ciudadanía y Globalización, PuntoRojo Libros, Sevilla, 2017, pp. 170-186.

TRIBUNAL DE JUSTICIA DE LA UNIÓN EUROPEA. Procedimiento prejudicial. Asunto C-71/14 East Sussex, ap. 52, y Asunto C 72/95 Kraaijeveld, ap. 56.

Disponible en: http://curia.europa.eu/juris/document/document.jsf;jsessionid=57DC4C 7460417E8F360B6ABAF35D5DCC text $=\&$ docid $=215512 \&$ pageIndex $=0 \&$ doclang $=$ es $\&$ mode $=$ req \&dir $=\& o c c=$ first $\&$ part $=1 \&$ cid $=260218$. Consulta en 06 de marzo de 2019.

UNECE. The United Nations Economic Commission for Europe. Convention on Access to Information, Public Participation in Decision-Making and Access to Justice in Environmental Matters.

UNIÓN EUROPEA. Carta de Derechos Fundamentales. Disponible en: http://www. europarl.europa.eu/charter/pdf/text_es.pdf. Consulta en: 06 de marzo de 2019.

UNIÓN EUROPEA. Diario Oficial de la Unión Europea (DOUE) C 275. 18.08.2017. Disponible en: https://eur-lex.europa.eu/legal-content/ES/ALL/?uri=OJ\%3AC\%3A2017 \%3A275\%3ATOC. Consulta en 06 de marzo de 2019.

UNIÓN EUROPEA. Diario Oficial de la Unión Europea (DOUE) L 156. 25.06.2003. Disponible en: https://www.boe.es/doue/2003/156/L00017-00025.pdf. Consulta en 06 de marzo de 2019.

UNIÓN EUROPEA. Diario Oficial de la Unión Europea (DOUE) L 264. 25.09.2006. Disponible en: https://www.boe.es/buscar/doc.php?id=DOUE-L-2006-81794. Consulta en 06 de marzo de 2019.

UNIÓN EUROPEA. Diario Oficial de la Unión Europea (DOUE) L 41. 14.02.2003. Disponible en: https://www.boe.es/doue/2003/041/L00037-00040.pdf. Consulta en 06 de marzo de 2019.

UNIÓN EUROPEA. Diario Oficial de la Unión Europea. Disponible en: https://eur-lex. europa.eu/legal-content/ES/ALL/?uri=OJ\%3AL\%3A2013\%3A354\%3ATO. Consulta en: 06 de marzo de 2019. 


\section{ACCESO A LA JUSTICIA EN MATERIA AMBIENTAL EN LA UNIÓN EUROPEA}

\section{Resumen}

La justicia ambiental constituye, en la Unión europea, el tercer pilar de garantía para los ciudadanos en la defensa de nuestro medio ambiente. Junto al acceso a la información, y la participación en materia ambiental, la justicia ambiental presenta un carácter dual: por un lado, el enjuiciamiento por los tribunales de justicia de todas aquellas materias, públicas y privadas, que puedan tener un impacto en las políticas ambientales; y por otro, la garantía de la legitimación ciudadana para impetrar acciones legales en defensa del medio ambiente, y ser, por tanto, parte legitimada en los procesos de esta naturaleza. en la Unión europea, la justicia ambiental, había quedado olvidada por hechos de diferente etología, hasta la aprobación en 2017 de la Comunicación de la Comisión relativa al acceso a la justicia en materia medioambiental, que vino a colmar esa laguna y dar carta de naturaleza a este derecho ciudadano tan relevante.

Palabras-clave: Justicia ambiental. Medio ambiente. Unión Europea. 


\title{
ACCESS TO ENVIRONMENTAL JUSTICE IN THE EUROPEAN UNION
}

\begin{abstract}
In the European Union, environmental justice is the third pillar of guarantee for citizens in the defence of our environment. Together with access to information and participation in environmental matters, environmental justice has a dual character: on the one hand, the prosecution by the courts of justice of all those matters, public and private, which may have an impact on environmental policies; and on the other hand, the guarantee of citizen legitimacy to take legal action in defense of the environment, and therefore be a legitimate party in processes of this nature. In the European Union, environmental justice had been forgotten due to facts of different ethology, until the approval in 2017 of the Commission's Communication on access to justice in environmental matters, which came to fill this gap and give a charter to this very relevant citizen's right.
\end{abstract}

Keywords: Environmental justice. Environment. European Union. 